\title{
Physics of arcing, and implications to sputter deposition
}

\author{
André Anders \\ Lawrence Berkeley National Laboratory, Berkeley, California, USA
}

\begin{abstract}
Arc and glow discharges are defined based on their cathode processes. Arcs are characterized by collective electron emission, which can be stationary with hot cathodes (thermionic arcs), or nonstationary with cold cathodes (cathodic arcs). A brief review on cathodic arc properties serves as the starting point to better understand arcing phenomena in sputtering. Although arcing occurs in both metal and reactive sputtering, it is more of an issue in the reactive case. Arcing occurs if sufficiently high field strength leads to thermal runaway of an electron emission site. The role of insulating layers and surface potential adjustment through current leakage is highlighted. In the situation of magnetron sputtering with "racetrack," the need for a model with two spatial dimensions is shown. In many cases, arcing is initiated by breakdown of dielectric layers and inclusions. It is most efficiently prevented if formation and excessive charge-up of dielectric layers and inclusions can be avoided.
\end{abstract}

Keywords: Arcing, sputtering, poisoned target, plasma processing and deposition

\section{Introduction}

Arcing on sputter targets and negatively biased substrates is known as one of the most challenging issues in physical vapor deposition of thin films and coatings. This is particularly true when high-rate deposition with reactive gases, large area deposition, and high power pulsed sputtering are considered. Much progress has been made in the development of power supplies that can handle arcing events with minimal damage to target and substrate. However, relatively little is known about the processes leading to arcs and the physics of the arcing events themselves. In this contribution, the issue of arcing is approached from the point of view of arc physics.

\section{Some Fundamentals of Arcs and Glows}

\subsection{Comparison of arcs and glows}

In some older textbooks, arcs are introduced as electrical discharges characterized by relatively high current (say, much greater than $1 \mathrm{~A}$ ) and low burning voltage (say, less than $50 \mathrm{~V}$ ). In contrast, glow discharges often have low current and high voltage. That description, of course, is a superficial and it will cause confusion when extreme cases are considered, such as high-power magnetron discharges as they are typical for today's large area deposition. Therefore, we need a more stringent approach to arcs and glows [1]. 
The fundamental difference between arcs and glows can be found in cathode processes. Arcs are characterized by a collective electron emission mechanism from the cathode, whereas glow discharge cathodes emit electrons by individual processes.

There are two fundamental modes of collective electron emission: thermionic and explosive. Correspondingly, one distinguishes between thermionic arcs and cathodic arcs.

In thermionic arcs, the most energetic electrons of the Fermi-Dirac distribution are able to overcome the potential barrier at the cathode surface, provided the cathode has a high temperature. Emission is often assisted by an electric field that deforms the potential barrier (Schottky effect). The current density can be described by the Richardson-Dushman equation,

$$
j_{\text {thermionic }}=A T^{2} \exp \left(-\frac{\phi}{k T}\right)
$$

where $A \approx 1.2 \times 10^{6} \mathrm{~A} \mathrm{~m}^{-2} \mathrm{~K}^{-2}$ is the universal Richardson constant, $\phi$ is the work function (the height of the potential barrier), and $T$ is the cathode surface temperature.

In contrast to thermionic arcs, cathodic arcs can operate with the cathode near room temperature. Electron emission occurs at hot, non-stationary, micron-size cathode spots. Research indicates that each cathode spot has an average current density of order $10^{12} \mathrm{~A} / \mathrm{m}^{2}$ and contains a self-organized cell structure. The highly nonlinear temperature relationship expressed in the Richardson-Dushman equation immediately points to a reason why microscopic spots form. To obtain higher electron current, it is energetically more effective to increase the temperature on a small area than to increase the area at constant temperature.

One may consider the operation of cathodic arc spots as a rapid sequence of microexplosions. In these explosive events, large amounts of electrons can overcome the potential barrier. The cathode material in the vicinity of the spot experiences phase transformations, ultimately resulting in fully ionized, rapidly expanding plasma. Thermal conduction in the solid increases the active spot area and reduces the power density, while the electrical conductivity below the spot is reduced at high temperature. Therefore, a microexplosion destroys its own favorable condition. As a side effect of plasma formation, material located between the dense plasma and the much colder cathode is in the liquid phase, subject to the pressure of the plasma. As a result, the material is ejected as droplets or "macroparticles," often under shallow angle to the cathode surface, and a crater is left on the cathode surface. The formation of macroparticles is highly undesirable and a major reason why arcing is of great concern to sputter deposition.

In both cases of thermionic and cathodic arcs, electrons are emitted in large amounts. It is impossible to emit only a few electrons via the mechanisms described. The emission is always a result of the collective behavior of many particles in a certain environment. In the case of cathodic arcs, there exists a minimum 'chopping' current and a minimum localized action. Collective electron emission is the key characteristics for an arc discharge.

Electron emission in glow discharges results from individual events, usually secondary electron emission by primary ion impact, however, also primary photons or electronically excited atoms can contribute. Each event deforms the potential barrier and provides energy that enables a discrete number of electrons to escape from the cathode surface. Obviously, 
the material, surface state, and energy and impact angle of the primary particle affect the outcome of each event.

Magnetron discharges are magnetically enhanced glow discharges, where the ionization probability of gas atoms or molecules by electron impact is greatly enhanced due to the motion of electrons in the ExB field. Although the plasma density is higher, and the operational pressure may be lower than in glow discharges without magnetic field, the cathode processes are still indicative for the glow nature of the discharge. Secondary electron emission by primary ions is only efficient when the impact energy exceeds about a couple of $100 \mathrm{~V}$, hence the glow discharge voltage is high, usually many $100 \mathrm{~V}$. Besides providing energy for secondary electron emission, primary ions bring enough energy to overcome the surface binding energy of atoms, leading to target sputtering, the desirable side effect that made us work in this field in the first place.

\subsection{Role of cathode surface conditions}

Surface contamination and non-metallic surface layers cause important modifications to the electron emission for both arcs and glows. It is clear that the potential barrier from the Fermi level to vacuum level is hidden by an additional potential structure as determined by the non-metallic material.

For glow and magnetron discharges, this is the well-known case of target poisoning. Arrival of an energetic ion at the poisoned target often results in a geater secondary electron current, although it is not clear whether this is due to tunneling enhanced by a nonmetallic layer or an effect of increased voltage and ion energy .

For arc discharges, phenomenology and parameters of cathode spots are greatly dependent on the presence of non-metallic layers and inclusions [2]. One generally distinguishes between type 1 and type 2 cathode spots. Type 1 occurs in the presence of non-metallic layers while type 2 burns on metal surfaces. Depending on the circumstances, type 1 and 2 may successively appear in the same arc discharge due to material erosion: the arc may start with type 1 spots and rather distinctly transitions into type 2 spots (hence the order of numbering). Type 1 spots produce small, separate craters, have relatively low erosion rate, and spot motion is fast. The plasma contains metal and non-metal species. In contrast, type 2 spots make chains of large craters, move slowly, and have high erosion rate. The plasma contains multiply charged metal ions of the cathode material.

\subsection{Thermal runaway and ignition of cathodic arc spots}

A key to understanding arc spot types, spot operation, characteristic times, and other parameters is to look at the life cycle of a spot, in particular the physics of spot ignition. Ignition of a spot occurs when the thermal run-away condition is fulfilled.

Thermal runaway may be explained as follows The cathode surface is subject to a high electric field. In the presence of plasma, most of the applied voltage drops in a very thin sheath near the cathode. Therefore, even a small voltage drop of order $10 \mathrm{~V}$, which is characteristic for arc discharges, can create an electric field at the cathode surface strong enough to cause field emission of electrons. Field emission current depends in a highly nonlinear way on the electric field strength. 
Since the surface is never perfectly flat and chemically uniform, there are always locations that produce an emission current density that is enhanced compared to the average. This enhancement sensitively depends on field-enhancement factors, the most imp ortant being geometric enhancement through nano-protrusions and step defects, and localized charges on insulating inclusions, e.g. grains of oxide, dust, flakes. From high-voltage experiments in vacuum it is known that the local field enhancement factor, $\beta$, which includes all types of enhancement, is often in the range 10-100, but in some extreme cases may exceed $10^{3}$. Therefore, there are conditions where the local electric field strength can be significantly higher than the average surface field strength. Coupling this field enhancement with the nonlinear field dependence of emission, one can easily see that electrons are emitted in a highly localized way.

That alone would not lead to spot formation. There exists a positive Eedback loop amplifying emission. The feedback is associated with localized ohmic heating of the emission site. A locally higher temperature increases the number of electrons in the distribution function that are able to overcome the potential barrier, which leads to even higher current density than provided via field emission. This higher current density in turn leads to greater ohmic heating of the site, which again increases electron emission. The temperature of the emission site may run-away and may quickly $(<1 \mathrm{~ns})$ exceed the melting and boiling temperature of the material. Phase transitions occur and dense plasma is formed, which expands into the available space on a slower time scale (10s of ns due to inertia). The spot is ignited.

The minimum local field required to ignite a spot depends on the material. For clean materials, the "cohesive energy rule" provides guidance on the "ease" of arcing, for example, aluminum $(3.4 \mathrm{eV} /$ atom $)$ is much easier to ignite than tantalum $(8.1 \mathrm{eV} / \mathrm{atom})$.

One should note that the combined field and temperature-based emission is known as thermofield emission, which is greater than the linear superposition of field and thermionic emission. Thermofield emission can approximated by [3]

$$
j_{T F}(T, \mathrm{E}) \approx k\left(A T^{2}+B \mathrm{E}^{9 / 8}\right) \exp \left[-\left(\frac{T^{2}}{C}+\frac{\mathrm{E}^{2}}{D}\right)^{-1 / 2}\right],
$$

where $\mathrm{E}$ is the electric field and A, B, C, and D, are material-specific constants.

The plasma emitted from an ignited spot expands and causes the sheath thickness nearby to contract since the sheath thickness depends in the plasma density. In the simplest case, when the magnetic field can be neglected and the electrode can be considered planar, the solution of the Poisson equation is Child's law, which can be written as

$$
s_{\text {Child }}=\frac{\sqrt{2}}{3} \lambda_{D e}\left(\frac{2 e|\Delta V|}{k T_{e}}\right)^{3 / 4} .
$$

where $\Delta V$ is the voltage drop in the sheath. The Child sheath thickness scales with plasma density because the electron Debye length $\lambda_{D e}=\sqrt{\varepsilon_{0} k T_{e} / e^{2} n_{e}}$ shrinks with increasing density (a typical plasma may have $\lambda_{D e} \approx 50 \mu \mathrm{m}$ and $s_{\text {Child }} \sim 1 \mathrm{~mm}$ ). A shrinking sheat h means that the cathode voltage drop has to be accommodated in less distance. The electric field strength increases, which may cause nearby electron emission centers to fulfill the thermal runaway condition, and hence new spots can be ignited. The plasma expands with 
$\sim 10^{4} \mathrm{~m} / \mathrm{s}$ from the spot and therefore a neighboring location in $10 \mu \mathrm{m}$ distance could see the plasma on a timescale of as little as $1 \mathrm{~ns}$.

An active (emitting) spot will increase its area in time ( 10-100 ns timescale) due to heat conduction, and thus the power density, which is $P \approx j \Delta V$, will be reduced in time because the current density, $j$, decreases. Additionally, the very hot cathode metal under the spot will have a higher resistance than the bulk metal, and therefore the potential of the spot surface will have a little less negative potential than the surrounding target area. For these reasons, a newly formed spot, electrically in parallel to the original spot, will take over the current from the original spot. The original spot will cool down and gradually $(\sim 1$ $\mu$ s timescale) cease to emit electrons.

These spot processes depend on the surface conditions [2]. The presence of a dielectric layer, or dielectric inclusions, promotes the buildup of surface charge and ignition of new spots, caus ing "premature death" of the parent spot. All other features of type 1 spots such as smaller craters and faster spot motion follow. In case of a well-eroded cathode, or otherwise cleaned metal surface, where type 2 spots are observed, dielectric charge enhancement is negligible, and geometric enhancements are more important. The most significant enhancement is produced by the spot action itself, namely protrusions developed at the crater rim. Consequently, ignition is most likely at the rim of an active crater. The time constants are related to the motion of cathode material and formation of a protrusion, which is slower than the characteristic time of plasma expansion. Therefore, type 2 spots produce chains of craters and have more time to "excavate" the cathode surface than type 1 spots.

\section{Arcing in Metal Sputter Deposition}

With the exception of some special cases of self -sputtering, metal deposition by sputtering is done using a noble gas such as argon. New targets usually have a thin dielectric layer (oxide), which is quickly removed when sputtering starts. Arcing is therefore only an issue when the current is high, i.e. when the sputter power settings are such that the sputter current exceeds the arc chopping current by a wide margin. This is in particular the case when high power pulsed magnetron sputtering (HPPMS) is employed [5]. In HPPMS, the current can readily exceed $100 \mathrm{~A}$, which is a current level normally used in arc deposition systems. At this level, it is diffic ult or impossible to rely on current signals for the determination of the arc or glow mode. This question can be best addressed by considering voltage. When arcing occurs, the voltage will sharply drop. At high current it is likely that spots quickly transition into type 2 . The plasma density is greater over the racetrack than outside due to ionization by magnetized electrons. Therefore, in the metal sputtering case, the sheath over the racetrack is thinner than outside the racetrack. The sheath voltage is the same at any point over the target.

A beautiful experimental demonstration of arcing on $\mathrm{Al}$ target in argon was presented by Tomasel and co-workers [6]. Using a high-speed camera, they caught images of arc spots ignited near the edge of the racetrack. The electrons emitted by the arc spot are magnetized in the field of the permanent magnets. One can see that even with noble gases, metals that easily form oxides have a non-metallic layer outside the racetrack, promoting arc ignition 
near the racetrack edge. This will be further discussed in the next section. The study is also a nice visual confirmation of the well-known $\mathbf{E x B}$ drift.

Wickersham and coworkers [7-9] demonstrated in a series of papers that dielectric inclusions such as $\mathrm{AbO}_{3}$ in an $\mathrm{Al}$ target cause arcing. They found that inclusions must have a critical size to reproducibly ignite an arc. Their findings could be interpreted in light of statements and models presented in the next section.

\section{Arcing in Reactive Sputter Deposition}

Arcing is a much greater issue in reactive sputter deposition than in metal deposition. Obviously, target poisoning and the buildup of a compound layer play an important role. If magnetron sputtering is considered, the main sputter area (the "racetrack") may remain free of a poisoned layer but a compound film always forms outside the racetrack. The arcing issue is especially severe when the compound is highly insulating, and it is further exacerbated when deposition is done with high current.

In the literature, different phenomenological arcing types have been distinguished, sometime labeled as flashover, unipolar arc, bipolar arc, and microarc (e.g. [10]). Obviously, not all arcing events are equal. From the arc physics point of view, it is tempting to search for a relation between these phenomenological types and arc spots of type 1 and 2 .

The timedependent behavior of an insulating layer on a sputter target was modeled by Barnat and $\mathrm{Lu}[11]$. The same model was also applied to study charging of insulating layers on biased substrates exposed to transient plasmas [12]. These authors used a 1dimensional equivalent circuit for the plasma-sheath-insulator-target structure (Fig. 1).

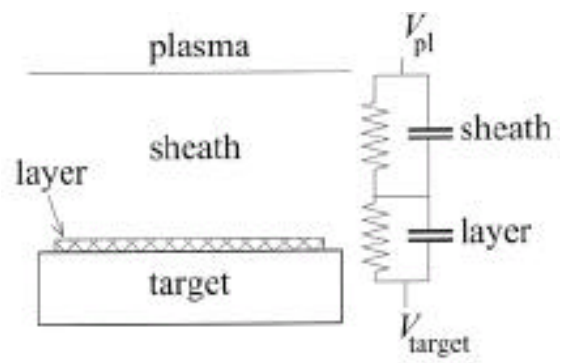

Fig. 1. Schematic for 1-dim model of sheath and layer between plasma and target, and their equivalent circuit.

The most important unknown is the surface potential of the insulator, $V_{\text {surf }}$. It results from the balance of charge (electrons, ions) accumulated via interaction with the plasma. The insulating layer can be considered as a planar capacitor with one electrode being the target surface. The sheath between insu lator surface and plasma is another capacitor. There may be a slowly varying current flowing, which can be taken into account by parallel resistors. By coupling ion fluid equations, the Poisson equation, and the equivalent circuit equations one can derive an expression for the time-dependent insulator surface potential, 


$$
\frac{d V_{\text {surf }}}{d t}=\frac{d V_{\text {target }}}{d t}+\frac{d_{\text {layer }}}{\kappa \varepsilon_{0}}\left(j_{i}+j_{e}+j_{\text {displace }}+j_{\text {leak }}\right),
$$

where $V_{t \text { arg et }}$ is the electrical potential of the sputter target, $d_{\text {layer }}$ is the thickness of the insulating layer, $\kappa$ is the dielectric constant of the insulating layer, and $\varepsilon_{0}$ is the permittivity of free space. The current densities in the bracket designate ion and electron currents from the plasma sheath, displacement current (negligible, unless very high frequencies are considered), and leakage current though the insulating solid layer. Note that some terms in the current balance are positive and others are negative.

If the layer has a noticeable conductivity, the leakage current is large and the layer surface potential will be close to the target potential. In the opposite case, when a highly insulating layer is formed, the leakage current can be neglected. In steady-state, the surface is floating, that is, its potential will adjust such as to precisely balance the flow of electrons and ions from the plasma. Since electrons are more mobile than ions, the surface potential will be negative with respect to the plasma potential, with the difference of a few $k T_{e}$, that is, of order 10 Volts. That implies that most of the voltage between target and plasma (several $100 \mathrm{~V}$ ) is actually dropping in the insulating layer, and the sheath voltage and thickness is reduced accordingly.

In the real world, the target is heavily sputtered in the racetrack area, and the insulating layer is mainly formed outside the racetrack. One has to consider different conditions over, near and far from the racetrack. Therefore, any realistic model of insulating layers on sputter targets should have at least two spatial dimensions.

The sheath above the different zones has to accommodate very different voltages: the full cathode fall over the racetrack and the floating potential over the insulating later. In contrast to the metal sputter case, the sheath thickness over the racetrack will be thicker than over the insulating layer. The transition zone will have a tilted sheath edge (Fig.2).

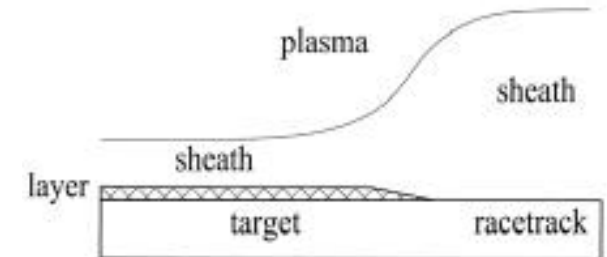

Fig. 2. Schematic of sheath structure at the racetrack edge.

Ions arriving at the transition zone have a non-normal angle of incidence and reduced current density, but their sputter yield is increased. In a recent study, Kim and Economou [13] developed a 2-dimensional sheath model for the metal-insulator transition. Although their voltage assumption was an order of magnitude smaller than what is observed in sputtering, their model is useful to improving our understanding arcing.

A very thin "poisoned" layer exists immediately, especially for metals that easily form compounds. As long as the layer is only a few monolayers thick, charge transport through the layer can occur for two reasons. One is the kinetic energy of the impacting ion, leading to local deformation and destruction of the film structure by creating a thermal spike that quenches in less than 1 ps. Such spikes can be studied with molecular dynamics 
simulations using environment dependent interaction potentials. The other reason is that even in the absence of thermal spikes, charge tunneling is likely. The tunneling mechanisms can involve electrons and holes, and depend on the material. Band-to-band tunneling has been identified as a dominant mechanism at high fields. For a very thin layer, net growth is the result of competition between deposition from scattered atoms and sputtering by ions accelerated in the sheath. Therefore, for some time, film growth outside the racetrack is slow or close to zero.

As the insulating layer slowly grows, tunneling eventually becomes greatly reduced and the penetration depth of primary ions becomes small compared to the layer thickness. Monte Carlo simulations using the T-DYN 4 code indicated a penetration depth of $3.2 \mathrm{~nm}$ for 500 $\mathrm{eV} \mathrm{Ar}^{+}$in $\mathrm{Al}_{2} \mathrm{O}_{3}$, thus subplantation of ions cannot explain leakage for thicker films. As the leakage current becomes smaller, the layer surface potential shifts to the floating potential. This will reduce bombardment by energetic ions and also set up a large field inside the layer. For example, if we consider an insulating film of $100 \mathrm{~nm}$ with a floating potential of about $-20 \mathrm{~V}$ with respect to the plasma potential, and a target potential of about $-500 \mathrm{~V}$ with respect to the plasma potential, the voltage drop in the layer is $480 \mathrm{~V}$, corresponding to about $4.8 \mathrm{~V} / \mathrm{nm}$ or $4.8 \times 10^{9} \mathrm{~V} / \mathrm{m}$. This extremely high field strength will cause the insulator to breakdown unless other leakage and loss mechanism still play a role. For example, the breakdown field strength for aluminum oxide in the thickness range of 23-187 $\mathrm{nm}$ has been determined to $0.9 \mathrm{~V} / \mathrm{nm}$ [14], 0.3-1.3 V/nm [15], and $0.74 \mathrm{~V} / \mathrm{nm}$ [16]. Ultrathin $\mathrm{Ta}_{2} \mathrm{O}_{5}$ films have a breakdown field of about $0.3 \mathrm{~V} / \mathrm{nm}$ [17].

When the layer surface potential approaches floating potential, ions will be much less accelerated than those ions over the racetrack. Therefore, there will be a bifurcation point in the layer growth process. Initially, insulating layer growth is very slow since ions sputter the growing layer. At the bifurcation point, the potential changes, which in turn increases net growth rate due to the lack of sputtering. That might explain the finite time of many minutes before arcing onset was observed for polished aluminum targets [18].

Additionally to vertical gradients, lateral potential gradients will develop, especially in the transition zone near the racetrack. Although the lateral field is weaker $\left(\sim 10^{7} \mathrm{~V} / \mathrm{m}\right)$, it can cause charge hopping on the surface, which in a two-dimensional model will appear as an additional leakage current term. The electric field is enhanced at the insulator-metal-gas triple point, which in the area of high voltage insulation is known to preferentially emit electrons, potentially developing into a flashover avalanche [19]. One may speculate that this kind of leakage is extremely frequent; it seems to correspond to Koski's [10] description of "microarcing" on insulators (in this context a somewhat misleading term). Occasionally, larger amounts of charge may be involved, giving rise to the observed "unipolar arcs" between insulator and target. Again, "flashover" is a more conventional term of this discharge phenomenon.

Additional leakage mechanisms could involve defects of the growing insulator, which may be caused by target roughness, dust particles, etc. This defect leakage current can result in avalanches through the layer, creating a highly conducting plasma channel. It will cause breakdown of the target voltage and onset of arcing (in Koski's nomenclature a "bipolar arc"), with all typical arc spot effects described earlier in this paper. In a rapid sequence, new arc spots will be ignited until the stored energy is exhausted or the power supply shuts down the arc. 
Segers and coworkers $[18,20]$ have experimentally shown that arcing in reactive sputtering occurs with very high probability in the transition zone next to the racetrack. They developed a one-dimensional model for the transition zone assuming that leakage through the insulator is present (therefore the layer surface will not reach the floating potential). In light of the transitional character of this zone, their model should be considered as a step towards a future, more comprehensive, 2-dimensional model.

\section{Arc Suppression Circuits and Other Mitigation Measures}

There are numerous approaches to mitigate and handle arcing events. In one approach, one tries to prevent the formation of a transition zone by moving either the target relative to the magnet (rotating magnetrons) or moving the magnets behind the target. In both cases, formation of a racetrack and transition zone is avoided (one has the additional benefit of better target utilization).

In another approach one neutralizes charge buildup by pulsing the target potential. In particular one may asymmetrically reverse the polarity of the applied voltage [21]. Good results have been obtained when the pulsing frequency was high and the pulse duty cycle moderate or low [22].

Yet another approach, related to the above, is dual magnetron sputtering. In this case, the target of a second magnetron serves as the anode of the first. Potential reversal can be symmetric, and one also solves the "disappearing anode" issue.

A last (but not least) line of defense is the use of "smart" power supplies. Arcs are detected by rising currents and/or falling voltage. As pointed out before, at very high sputter power levels, voltage drop detection is much less ambiguous than current changes. Once detected, a smart power supply shuts down the arc by interrupting energy supply, and in some cases the voltage is reversed. Sputter conditions are restored after the arc spot region was allowed to cool down. Developers of power supplies have succeeded in reducing the duration between arc detection and shutdown to some $10 \mu \mathrm{s}$. However, since arc ignition and spot development are sub-microsecond events, residual damage may still occur, such ejection of some macroparticles.

\section{Conclusions}

Arcing phenomena on sputter targets can be related to the physics of arc phenomena which were mainly investigated for other applications, such as arc switching, high voltage insulation, and cathodic arc deposition. Arc spot types 1 and 2 are associated with the presence of non-metallic surface layers, and this can be related to arcing phenomena seen in metal and reactive sputtering, respectively. Arcing is an issue especially in reactive deposition. The ignition of arc spots is promoted by the presence of thin insulating films and inclusions. As the leakage current is reduced, the surface potential of insulating layers approaches the floating potential, and therefore most of the applied target potential drops in the insulating compound. As a result, insulating film growth accelerates due to reduced sputtering, but the high field strength can be sufficient to cause dielectric br eakdown, which initiates an arc discharges. Mitigation measures focus on the prevention of the formation of insulating layers near the racetrack, the prevention of charge buildup by frequent reversal of 
polarity, and smart power supplies that can rapidly shut down arcs, thereby minimizing damage to the substrate.

\section{Acknowledgments}

Helpful discussions with Bill Sproul and other friends and colleagues are gratefully acknowledged. This work was supported by the Assistant Secretary for Energy Efficiency and Renewable Energy, Office of Building Technology, of the U.S. Department of Energy under Contract No. DE-AC03-76SF00098.

\section{References}

[1] A. Anders, Cathodic Arc Plasma Deposition (in preparation). New York: Springer, 2005.

[2] B. Jüttner, Physica 114C (1982) 155.

[3] E. Hantzsche, Beitr. Plasmaphys. 22 (1982) 325.

[4] E. Hantzsche, Beitr. Plasmaphys. 17 (1977) 65.

[5] V. Kouznetsov, K. Macak, J. M. Schneider, et al., Surf. Coat. Technol. 122 (1999) 290.

[6] F. G. Tomasel, D. Carter, H. Walde, et al., Plasma Sources Sci. Technol. 12 (2003) 139.

[7] C. E. Wickersham Jr., J. E. Poole, A. Leybovich, and L. Zhu, J. Vac. Sci. Technol. A19 (2001) 2767.

[8] C. E. Wickersham Jr., J. E. Poole, J. S. Fan, and L. Zhu, J. Vac. Sci. Technol. A 19(2001) 2741.

[9] C. E. Wickersham Jr., J. E. Poole, and J. S. Fan, J. Vac. Sci. Technol. A 20 (2002) 833.

[10] K. Koski, J. Hölsä, and P. Juliet, Surf. Coat. Technol. 115 (1999) 163.

[11] E. V. Barnat and T. M. Lu, J. Appl. Phys. 90 (2001) 5898.

[12] Z. L. Dai and Y. N. Wang, Surf. Coat. Technol. 165 (2003) 224.

[13] D. Kim and D. J. Economou, J. Appl. Phys. 94 (2003) 2852.

[14] H. J. De Wit and C. Crevecoeur, Phys. Lett. A 50 (1974) 365.

[15] N. Klein, J. Appl. Phys. 53 (1982) 5840.

[16] T. Seino and T. Sato, J. Vac. Sci. Technol. A 20 (2002) 634.

[17] J.-Y. Kim, M. C. Nielsen, E. J. Rymaszewski, and T.-M. Lu, J. Appl. Phys. 87 (2000) 1448.

[18] A. Segers, D. Depla, K. Eufinger, et al., "Reactive sputtering," Nouvelles Tendances en Procédés Magnetron et arc pour le Dépot de Couches Minces, Gent, Belgium, 2003, 25-39.

[19] R. V. Latham, High Voltage Vacuum Insulation. London and New York: Academic Press, 1981.

[20] A. Segers, N. V. Bekaert, D. Depla, et al., "Arc discharge in the reactive sputtering of electrical insulating compounds," Society of Vacuum Coaters, 45th Annual Technical Conference Proceedings, Orlando, FL, 2002, 30-35.

[21] J. Sellers, Surf. Coat. Technol. 98 (1998) 1245.

[22] A. Belkind, Z. Zhao, D. Carter, et al., "PulsedDC reactive sputtering of dielectrics: pulsing parameter effects," Society of Vacuum Coaters, 43th Annual Technical Conference Proceedings, Denver, CO, 2000, 86-90. 